\title{
CONSUMO DE PSICOFÁRMACOS ENTRE ALUNOS DE MEDICINA DO PRIMEIRO E SEXTO ANO DE UMA UNIVERSIDADE DO ESTADO DE SÃO PAULO
}

Ilanna Sobral de Luna, Angelica Augusta Grigoli Dominato, Flávia Ferrari, Airan Lobo da Costa, Andressa

Christhinie Pires, Gerson da Silva Ximendes

Universidade do Oeste Paulista - UNOESTE, Curso de Medicina, Presidente Prudente, SP. E-mail: ilaannasobral@hotmail.com

\section{RESUMO}

O objetivo do estudo foi avaliar a diferença no uso de drogas psicoativas entre os alunos do primeiro e sexto ano do curso de medicina de uma universidade do interior paulista, identificando os fatores associados ao uso, compreendendo os fatores precipitantes e a ciência dos riscos de dependência. É um estudo transversal de cunho quantitativo com a aplicação de questionário. Dos entrevistados do primeiro ano, $23 \%(n=23)$ afirmaram fazer uso de algum tipo de psicofármaco durante o período de coleta dos dados. Enquanto dos entrevistados do sexto ano, $50 \%(n=50)$ afirmaram fazer o uso de psicofármacos no período em que os dados foram coletados. Foi demonstrado que os estudantes de medicina dos últimos anos utilizam mais psicofármacos do que os estudantes que iniciaram o ingresso evidenciando a influência do curso sobre a medicalização.

Palavras-chaves: psicotrópicos, estudantes de medicina, educação médica, medicalização, educação superior.

\section{CONSUMPTION OF PSYCHOTROPIC DRUGS AMONG THE FIRST MEDICAL STUDENTS AND SIXTH YEAR OF A UNIVERSITY IN SÃO PAULO}

\begin{abstract}
The objective of the study was to evaluate the difference in psychoactive drug use among students of the first and sixth year of the medical course of a university in the interior of São Paulo, identifying the biopsychosocial factors associated with the use, including precipitating factors and the science of the risks of dependency. It is a cross-sectional quantitative study with the application of a questionnaire. Of the firstyear interviewees, $23 \%(n=23)$ reported using some form of psychotropic medication during the data collection period. While the sixth years interviewed, $50 \%(n=50)$ reported using psychotropic drugs at the time the data were collected. It has been shown that medical students in recent years use more psychoactive drugs than the students who started admission, showing the influence of the course on medicalization.
\end{abstract}

Keywords: psychotropic drugs, undergraduate students, medical education, medicalization, college education.

\section{INTRODUÇÃO}

Os psicofármacos estão cada dia mais sendo utilizados por adolescentes, devido a diversos motivos, nos quais os fatores biopsicossociais merecem destaque ${ }^{1}$. Identificar a realidade do uso de psicoativos entre estes indivíduos, através da aplicação de um questionário, reunindo dados epidemiológicos acerca do assunto, é uma importante ferramenta para formulação e avaliação de políticas públicas adequadas e destinadas à prevenção $0^{2,3}$.

As consequências do uso de substâncias psicoativas variam de acordo com o tipo, quantidade, interação da substância utilizada e 
apresenta uma evolução entre adolescentes quando comparados aos adultos ${ }^{4}$. O princípio ativo destes fármacos possui potencial de gerar dependência e reações adversas como a alteração do comportamento, cognição, sono, apetite e alteração das funções motora e autônoma de quem os consome ${ }^{5}$. Então, o uso indiscriminado dos medicamentos controlados é um problema de saúde pública. A utilização prolongada dos psicofármacos, além de efeitos colaterais indesejáveis, dependência química podem também gerar dificuldades quanto ao término do tratamento ${ }^{9}$. De acordo com a Organização Mundial de Saúde o abuso de substâncias psicoativas por jovens brasileiros inclui a mortalidade entre as dez mais altas do mundo na faixa etária de 15 a 25 anos $^{6}$.

O fenômeno atual do abuso de medicamentos psicotrópicos reflete também no cenário universitário, onde os antidepressivos, ansiolíticos, antimaníacos, antipsicóticos, psicoestimulantes entre outras estão presentes com aumento progresso. Isso demanda de um melhor conhecimento a respeito da problemática, que engloba uma série de aspectos biopsicossociais, que cerceiam 0 mundo acadêmico ${ }^{6}$.

Na contemporaneidade o ritmo e estilo de vida levam a população a vivenciar situações cada vez mais estressantes e difíceis ${ }^{7}$. Cobranças por produtividade, trânsito intenso, excesso de atividades, balbúrdia, entre outras, levam as pessoas à busca de soluções para contornar a ansiedade decorrente destas vivências. Uma das opções adotadas refere-se ao uso de substâncias psicoativas, para dormir, para obter um melhor rendimento em atividades cotidianas, assim como nos estudos ${ }^{8}$.

O uso de psicoestimulantes por jovens estudantes tem sido uma preocupação global, nos países como Canadá, Estados Unidos e Inglaterra esse assunto vem sendo bastante discutido após a realização de estudos epidemiológicos que indicaram o aumento do uso destes medicamentos pelos universitários para melhorar o rendimento acadêmico ${ }^{10,11}$.

Mundialmente, os medicamentos destinados ao tratamento de doenças mentais ocupam o 9o lugar dentre os mais vendidos, representando cerca de $7 \%$ das vendas. Dentre aqueles psicofármacos mais dispensados, no mundo, estão os benzodiazepínicos ${ }^{12}$.
Em contrapartida, um composto estimulante do sistema nervoso central da classe das anfetaminas, o Cloridrato de Metilfenidato, vem sendo utilizado para o tratamento do déficit de atenção e narcolepsia. No entanto, observa-se um aumento do seu uso entre os estudantes sem indicação de uso médico. Fato este que preocupa as autoridades no assunto, especialmente pela forma ilegal de aquisição do mesmo. Segundo a convenção da ONU de 1971, quando o Brasil assumiu o compromisso do controle na dispensação do referido fármaco, é necessário um receituário especial para adquiri-lo, pois apresenta risco de abuso e dependência ${ }^{13,14}$.

Desse modo, o presente estudo objetiva avaliar a diferença no uso de drogas psicoativas entre os alunos do primeiro e sexto ano do curso de medicina de uma universidade do interior paulista, identificando as causas associadas ao uso, compreendendo os motivos precipitantes e a ciência dos riscos de dependência.

\section{METODOLOGIA}

Realizou-se um estudo de caráter descritivo e exploratório do tipo transversal. A população em estudo foi constituída de 200 estudantes matriculados no curso de medicina de uma universidade. A amostra, por sua vez, foi formada por 100 alunos do primeiro ano do curso e 100 alunos do sexto ano.

Os dados foram coletados por meio de um questionário fechado composto por duas partes. Na primeira parte do questionário foram coletadas informações sócio-demográficas e na segunda parte o objetivo foi coletar informações sobre aquisição e utilização do medicamento como frequência de uso, obtenção da receita médica, tipo de medicamento, fatores envolvidos com uso da droga e a ciência dos efeitos adversos. A coleta de dados ocorreu entre os meses de maio e junho de 2017. Foram respondidos 200 questionários entre alunos abordados aleatoriamente e que aceitaram participar da pesquisa.

Para a coleta dos dados foi empregada a técnica de autorrelato estruturado. As informações eram registradas pelos sujeitos (autopreenchimento) e para análise dos dados, foi utilizada abordagem quantitativa. Após a codificação de cada uma das variáveis, criou-se um dicionário de dados para a construção de um banco de dados eletrônico no programa MS Excel (Windows ${ }^{\circledR}$ ). O projeto foi aprovado pelo Comitê 
de Ética e Pesquisa em Humanos da Universidade do Oeste Paulista sobre o Protocolo núm. 3693. Os participantes assinaram o termo de consentimento livre e esclarecido, de acordo com a Resolução do Conselho Nacional de Saúde (CNS 466/12).

\section{RESULTADOS}

Dos 200 estudantes que participaram do estudo, 100 estavam regularmente matriculados no primeiro ano do curso de medicina e 100 estavam matriculados no sexto ano. Os dados de sexo e idade dos participantes encontram-se na Tabela 1, mostrando um número maior de participantes do sexo feminino em ambos os anos do estudo, com uma idade média, no primeiro ano de 21 anos (com amplitude de 17 . 40 anos) e no sexto ano de 27 anos (variando entre 22 - 47 anos).

Tabela 1. Variáveis sexo e idade média dos participantes do 1ㅇ e 6을 anos do curso de medicina.

\begin{tabular}{lcccccc}
\hline & \multicolumn{2}{c}{ 10 ano } & \multicolumn{2}{c}{ 60 ano } & \multicolumn{2}{c}{ Total } \\
\hline Idade (anos) & \multicolumn{2}{c}{21} & \multicolumn{2}{c}{27} & & \\
Sexo & $\mathrm{n}$ & $\%$ & $\mathrm{n}$ & $\%$ & $\mathrm{n}$ & $\%$ \\
Feminino & 66 & 66 & 51 & 51 & 117 & 58,5 \\
Masculino & 34 & 34 & 49 & 49 & 83 & 41,5 \\
Total & 100 & 100 & 100 & 100 & 100 & 200 \\
\hline
\end{tabular}

Dos entrevistados do primeiro ano, $23 \%$ $(n=23)$ afirmaram fazer uso de algum tipo de psicofármaco durante o período de coleta dos dados, desses $70 \%$ são do sexo feminino. Entretanto, dentre os entrevistados do sexto ano, $50 \% \quad(n=50)$ afirmaram fazer 0 uso de psicofármacos no período em que os dados foram coletados, sendo que, $62 \%$ desses são do sexo masculino.

Em relação ao período de uso desses medicamentos pelos estudantes do primeiro ano, a média de tempo encontrada foi de 20 meses, enquanto que para os estudantes do sexto ano foram 29 meses. A maioria (65\%) dos participantes do primeiro ano alegaram obter a receita do fármaco em uso através de consulta médica, $22 \%$ com amigos ou familiares e $13 \%$ conseguiu de forma ilegal. Dos alunos do sexto ano $64 \%$ afirmaram obter a receita por meio da consulta médica e $36 \%$ através de amigos ou familiares.

Além disso, os dados coletados evidenciaram que a classe dos psicoestimulantes foram os medicamentos mais utilizados pelos estudantes de medicina do primeiro e sexto ano, sendo respectivamente, $65 \%$ e $34 \%$. Enquanto que os antidepressivos representaram a segunda classe mais relatada, sendo $30 \%$ pelos voluntários do primeiro ano e $32 \%$ para aqueles do sexto ano.

Dentre os principais fatores relatados, para o uso dos medicamentos estudados, $48 \%$ dos participantes do primeiro e $62 \%$ do sexto ano apontaram o curso, como principal fator desencadeador. Outros fatores foram relatados por $39 \%$ dos voluntários do primeiro ano e $30 \%$ pelos do sexto ano. Ademais, 91\% dos participantes do primeiro ano da pesquisa, usuários de psicofármacos afirmaram ter consciência dos efeitos colaterais, malefícios e riscos de dependência advindos do seu consumo e aumentando para a totalidade dos voluntários com consciência para seus riscos. A Tabela 2 evidencia a comparação dos resultados obtidos sobre o sexo, tempo e tipo de medicamento em uso e forma de aquisição, entre os voluntários do 1 ㅇ e 6 o ano. 
Tabela 2. Informações sobre as variáveis sexo, tempo médio de uso, forma de obtenção do medicamento, classe farmacológica, fatores precipitantes e consciência sobre os riscos de uso dos de psicofármacos, entre os participantes do 1 으 e 60 ano.

\begin{tabular}{lcc}
\hline Variáveis & 10 ano & 60 ano \\
\hline Sexo & $\%$ & $\%$ \\
Masculino & 30 & 62 \\
Feminino & 70 & 38 \\
\hline Tempo médio de uso & 20 meses & 29 meses \\
\hline Obtenção do fármaco & & \\
Receita médica & 65 & 64 \\
Amigos e familiares & 22 & 36 \\
Sem prescrição & 13 & 0 \\
\hline Classe farmacológica & & \\
Psicoestimulantes & 65 & 34 \\
Antidepressivos & 30 & 32 \\
Ansiolíticos & 5 & 34 \\
\hline Fatores precipitantes & & \\
Cursar medicina & 48 & 62 \\
Outros fatores & 39 & 30 \\
Familiares & 13 & 6 \\
Profissionais & 0 & 2 \\
\hline Consciência dos riscos & & \\
Sim & 91 & 100 \\
Não & 9 & 0 \\
\hline
\end{tabular}

\section{DISCUSSÃO}

No Brasil, o ingresso no curso superior tem sido estimulado por programas de incentivo educacional, mantidos pelo governo federal contribuindo com o aumento da faixa etária entre os estudantes, como foi observado na pesquisa. Em geral, as mulheres são a maioria entre os alunos, desta forma, no presente trabalho, detectou-se que um número maior entre os estudantes do primeiro ano ao contrário da realidade do último ano. Então os autores descreveram que a faixa etária estava entre 20 a 49 anos e $52 \%$ de mulheres ${ }^{3}$. Entretanto, em outro estudo a média de idade encontrada foi de 22 anos, porém com variações entre 20,5 a 23,5 anos de idade e $68 \%$ eram do sexo feminino ${ }^{5}$.

A vida acadêmica dos estudantes universitários exige dedicação de muitas horas de estudos, especialmente no curso de medicina, que se tornam momentos extenuantes. Os estudos encontrados na literatura descrevem que além da graduação, a rotina médica é considerada atividade de alta pressão e tensão psicológicas, influenciando no desempenho do estudante. Muitos jovens, então, para suportar o estresse e cansaço utilizam medicamentos controlados para atenuar a ansiedade e preocupação gerada. Em uma avaliação geral, foi possível constatar que os estudantes de medicina do sexto ano utilizam mais psicofármacos, quando comparados aos estudantes do primeiro ano, demonstrando que a evolução do curso tem íntima relação com o uso desses compostos medicamentosos, aliados a não demonstração de insegurança, cansaço, tristeza além de responder às exigências acadêmicas, da sociedade e dos colegas. Por isso, a imposição de ritmos de vida intenso podem comprometer a qualidade de vida do estudante ${ }^{15}$. Em outros estudos direcionados ao uso de medicamentos psicoativos estão relatados que $18 \%$ dos estudantes fizeram uso de psicoestimuolantes (anfetaminas e metilfenidato) pelo menos uma vez na vida, sendo que $60 \%$ desses relataram utilizar essa classe de medicamentos durante o curso de medicina ${ }^{11}$. Em duas escolas de medicina na Lituânia $8,1 \%$ dos estudantes relataram fazer uso de intensificadores do cognitivo pelo menos uma vez na vida, sendo que os homens utilizaram mais que as mulheres $(14,6 \% \text { e } 5,1 \%)^{11}$. E ainda, $17 \%$ 
dos entrevistados utilizaram medicamentos simpatomiméticos, sem indicação médica, e 79\% fizeram uso no último ano ${ }^{14}$.

No decorrer do curso o estresse dos estudantes está aumentado devido à sobrecarga acadêmica, conflitos interpessoais e emocionais como as situações de óbito e sofrimento, vivenciados, sobre tudo, pelos últimos anos do curso, período de internato, quando mantêm contato direto e contínuo com os pacientes. Esses fatores podem comprometer a qualidade de vida gerando como consequência dificuldades em relacionamentos sociais e o abuso de substâncias como os psicofármacos ${ }^{16}$. Nos estudos encontrados na literatura demonstraram que as razões para o uso de compostos para a melhora no desempenho cognitivo apresentaram a melhoria da concentração, aliada à memória e desempenho na academia, aumento do tempo de estudo e estado de alerta, experimentação e ainda a influência dos amigos ${ }^{14}$. O uso de psicoestimulantes para $32 \%$ dos estudantes obtiveram a melhoria da concentração e $23 \%$ usavam para manterem-se em estado de alerta, $18 \%$ relataram melhora no desempenho na academia, entre outras razões como o aumento da energia (18\%), usos em festas ou recreação (5\%), perda de peso $(1 \%)^{16}$. Ainda, como um auxiliar para o estudo (69\%) e aumento da concentração $(65 \%)^{14}$.

Uma grande preocupação para as autoridades de saúde é a forma de obtenção dos medicamentos psicoativos, uma vez que a receita médica nem sempre representa a realidade e necessidade de uso de medicamentos como para o TDAH (transtorno do déficit de atenção e hiperatividade), ou de antidepressivos, entre outros medicamentos. Na pesquisa foi observado que a maioria dos participantes do primeiro ano alegaram obter a receita do fármaco em uso através de consultas médicas, seguido daqueles medicamentos obtidos com amigos ou familiares e finalmente a obtenção sem prescrição. Os voluntários do sexto ano obtiveram as receitas através da consulta médica e por amigos ou familiares. Os dados revelam que a aquisição dos medicamentos através de amigos ou familiares, apesar de não ser a principal fonte, demonstram a automedicação, ou seja, sem a indicação de profissional habilitado, acarretando em medicalização descontrolada, excessiva e por vezes, desnecessária pelo estudante ${ }^{17,18}$. Em outro estudo destacou-se o estímulo de uso através dos amigos e colegas de turma (44\%), outras fontes (37\%), familiar com diagnóstico de TDAH $(4,9 \%)^{19}$. O uso não médico dos psicoestimulantes ainda foi detectado em 63\% dos quando adquiridos por amigos, parentes, colegas de classe ou conhecidos ${ }^{20}$.

Sobre a frequência de uso de medicamentos psicoativos se destacou a classe dos psicoestimulantes como os mais utilizados entre os voluntários do primeiro e sexto ano, seguida pelos antidepressivos. Então, para que consigam otimizar o estudo, os voluntários universitários, utilizam os psicoestimulantes, sobretudo pelo volume de assuntos e disciplinas pertencentes à estrutura curricular do curso de medicina. Aliado a isso encontra-se a aceleração da informação em decorrência das tecnologias. Fundamentando também, os resultados apresentados sobre os fatores envolvidos com uso dessa medicação, visto que os alunos do primeiro (48\%) e sexto (62\%) ano apresentaram o curso, como principal fator ${ }^{19}$. Os antidepressivos como segunda classe mais frequente justifica-se, pois, os sintomas depressivos são prevalentes na população universitária, sendo relatado em pesquisas brasileiras, demonstrando uma prevalência de sintomas depressivos em $27 \%$ dos estudantes de medicina pesquisados ${ }^{20,21}$.

A ocorrência dos efeitos colaterais, maléficos e riscos de dependências após o uso dos medicamentos é popular entre os estudantes da pesquisa. Constatação preocupante, uma vez que, apesar da maioria dos estudantes usuários terem consciência sobre os riscos, eles submetem-se ao uso mesmo sem o acompanhamento profissional necessário. Então, a presença das comorbidades como no diagnóstico de doença psiquiátrica (9\%) e doença cardiovascular (3\%) fazem parte da realidade desses usuários ${ }^{22}$.

Conclui-se assim que os estudantes de medicina dos últimos anos utilizam mais psicofármacos do que os estudantes que iniciaram o ingresso evidenciando a influência do curso sob a medicalização. Além disso, foi observado que a classe dos psicoestimulantes é a mais utilizada, o curso médico é o principal fator precipitante do uso dos fármacos e que a maioria dos estudantes têm noção dos riscos à saúde causados pelo consumo. Ademais, novos estudos devem ser realizados em escolas médicas e da área de saúde, compreendendo maior número de universitários. 


\section{CONFLITO DE INTERESSES}

Os autores declararam não haver qualquer potencial conflito de interesse que possa interferir na imparcialidade deste trabalho cientifico.

\section{REFERÊNCIAS}

1. Abi-Ackel MM, Lima-Costa MF, Castro-Costa E, Loyola Filho AL. Uso de psicofármacos entre idosos residentes em comunidade: prevalência e fatores associados. Rev Bras Epidemiol. 2017;20(1):57-69. DOI: http://dx.doi.org/10.1590/19805497201700010005

2. Moura DCN, Pinto JR, Martins P, Arruda Pedrosa K, Carneiro MGD. Uso abusivo de psicotrópicos pela demanda da Estratégia Saúde da Família: revisão integrativa da literatura. SANARE Rev Polít Púb. 2017;15(2):136-44.

3. Martins KS. Associação entre estilos parentais e consumo de drogas em adolescentes. [Dissertação]. Mestrado em Psicologia. Universidade Federal de Santa Catarina, 2016. Diponível em: https://repositorio.ufsc.br/bitstream/handle/1234567 89/172584/343610.pdf?sequence $=1$

4. Cruz MT, Cruz EL, Torres JRP. Avaliação do uso de medicamentos psicotrópicos pelos pacientes da farmácia municipal de Terra Roxa D'oeste/PR. Rev Thêma Scientia. 2016;5(1):131-7.

5. Martínez GI, Martínez LM, Rodríguez MA, Mesa PA, Muñoz JE, Bedoya J. Características del consumo de fármacos psicotrópicos en estudiantes de Ciencias de la Salud. Vitae. 2008;15(2).

6. OMS. Neurociência do consumo e dependência de substancias psicoativas. 2004. Disponível em: www.who.int/substance_abuse/publications/en/Neur oscience_P.pdf

7. Silva $E A B$. Plano de ação com vistas à redução do uso de medicamentos psicotrópicos e melhorar o estilo de vidas de pacientes com doenças mentais. [Monografia]. Especialização em Atenção Básica em Saúde da Família. Universidade Federal de Minas Gerais, 2017. Diponível em: https://ares.unasus.gov.br/acervo/bitstream/handle/ ARES/8847/plano-acao-vistas-reducao-usomedicamento. pdf?sequence $=1$ \&isAllowed $=y$

8. Santos LP, Oliveira AA, Salvi JO. Farmacovigilância de medicamentos psicotrópicos no município do Vale do Paraíso, RO. Rev Cient FAEMA. 2015;6(2):36-48.

9. Neto CDP, Martins ÁKL, de Oliveira FB, de Sousa Leite E, Pimenta CJL, de Oliveira Bezerra ML. Consumo de benzodiazepínicos entre idosos na estratégia saúde da família: revisão integrativa. Rev Enferm UFPE. 2016;10(12):4646-56. DOI:
10. Couto FR. Uso de psicotrópicos em policiais militares. Ciên Saúde Col. 2015;20(8):2499-2510. DOI: http://dx.doi.org/10.1590/141381232015208.15152014

11. Nascimento MO, Micheli D. Avaliação de diferentes modalidades de ações preventivas na redução do consumo de substâncias psicotrópicas em estudantes no ambiente escolar: um estudo randomizado. Ciênc Saúde Col. 2015;20(8):2499-2510. DOI: $\quad$ https://doi.org/10.1590/141381232015208.15152014

12. Soares J. O uso de medicamentos controlados por estudantes do curso de graduação em Odontologia na Universidade Federal de Santa Catarina. [Monografia]. Universidade Federal de Santa Catarina, Florianópolis, 2017. Disponível em:

https://repositorio.ufsc.br/bitstream/handle/1234567 89/176431/TCC\%20corrigido\%20.pdf?sequence=1

13. Walylo A, Pulcinelli LF, Oliveira LA, Flores CM. Avaliação das prescrições médicas de Ritalina ${ }^{\circledR}$ (metilfenidato) $10 \mathrm{mg}$, disponibilizadas no serviço público de saúde no Município de Ponta Grossa, durante o período de janeiro à junho de 2015. [Monografia]. Faculdade Sant'ana, São Paulo, 2017. Disponível em: http://iessa.edu.br/revista/index.php/tcc/article/dow nload/87/34

14. Medeiros JM, Barbosa AG. Uso não prescrito de cloridrato de metilfenidato entre estudantes universirários. Rev Pesqui Interdiscip. 2017;2(2):51424. DOI: http://dx.doi.org/10.24219/rpi.v2i2.275

15. Feodrippe ALO, Brandão MCF, Valente TCO. Medical students' quality of life: a review. Rev Bras Educ Med. 2013;37(3):418-28. DOI: http://dx.doi.org/10.1590/S0100-

55022013000300014

16. Wanscher D, Prado GP, Frigo J. Uso de psicotrópicos por alunos do ensino superior. Rev UNINGÁ. 2018;18(2):5-9.

17. Cruz PS, Caramona M, Guerreiro MP. Uma reflexão sobre a automedicação e medicamentos não sujeitos a receita médica em Portugal. Rev Port Farmacoter. 2015;7(2):83-90.

18. Castro C, Martins J, Nunes M, Sousa F, Antão C. A Automedicação nos alunos da Escola Superior de Saúde do Instituto Politécnico de Bragança. Millenium J Fdifration Technol Health. 2016;1:123-30.

19. Silveira VI, Oliveira RJF, Caixeta MR, Padua Andrade BB, Costa RGL, Santos GB. Uso de psicoestimulantes por acadêmicos de medicina de uma universidade do sul de Minas Gerais. 
2015;13(2):186-92.

DOI:

http://dx.doi.org/10.5892/ruvrd.v13i1.239

20. Emanuel RM, Frellsen SL, Kashima KJ, Sanguino SM, Sierles FS, Lazarus CJ. Cognitive enhancement drug use among future physicians: findings from a multi-institutional census of medical students. J Gen Intern Med. 2013;28(8):1028-34. DOI: http://dx.doi.org/ 10.1007/s11606-012-2249-4

21. Lengvenyte A, Strumila R, Grikiniene J. Use of cognitive enhancers among medical students in Lithuania Nordic Studies Alcohol Drugs. 2016;33(2):173-88.

DOI:

https://doi.org/10.1515/nsad-2016-0014

22. Retief $M$, Verster C Prevalence and correlates of non-medical stimulants and related drug use in a sample of South African undergraduate medical students. South African J Psych. 2016;22(1):a795. DOI: http:// dx.doi.org/10.4102/sajp. v22i1.795

Recebido para publicação em 24/02/2018

Revisado em 22/04/2018

Aceito em 07/05/2018 